\title{
COMPARISON OF LINEAR MULTIVARIABLE, PARTIAL LEAST SQUARE REGRESSION, AND ARTIFICIAL NEURAL NETWORK ANALYSES TO STUDY THE EFFECT OF DIFFERENT PARAMETERS ON ANODE PROPERTIES
}

\author{
Dipankar Bhattacharyay $^{1}$, Duygu Kocaefe ${ }^{1}$, Yasar Kocaefe ${ }^{1}$, BrigitteMorais ${ }^{2}$ \\ ${ }^{1}$ University of Quebec at Chicoutimi; 555 Boulevard de l'Université, Chicoutimi, QC, G7H 2B1, Canada \\ ${ }^{2}$ Aluminerie Alouette Inc.; 400, Chemin de la Pointe-Noire, Sept-Îles, QC, G4R 5M9, Canada
}

Keywords: Artificial neural network, linear multivariable analysis, partial least-square analysis, carbon anodes, data analysis

\begin{abstract}
Carbon anodes constitute a substantial part of the cost during the electrolytic production of aluminum. The industry tries to minimize the consumption of anodes by improving their quality. Therefore, a clear understanding of the impact of the quality of raw materials as well as process parameters on anode properties is important.
\end{abstract}

The plants have a large collection of data, which is complex and difficult to analyze using conventional methods. In this article, linear multivariable (LMA), partial least square regression (PLS), and artificial neural network (ANN) analyses are presented and compared as tools to predict the influence of different parameters on anode property. Published data have been processed using Matlab software to carry out the analyses. The results clearly show that ANN is the best tool for prediction purposes. Unlike other methods, ANN can handle nonlinear complex relations even if a well-defined relationship is not available.

\section{Introduction}

Cost of carbon anodes make up an important part of the total cost of the electrolytic production of primary aluminum. Aluminum oxide is reduced to aluminum with the use of carbon in anodes, and carbon is converted to carbon dioxide gas during this process. The theoretical carbon consumption is $0.334 \mathrm{~kg} \mathrm{C} / \mathrm{kg} \mathrm{Al}$. However, the actual consumption is higher than the theoretical value due to side reactions and current efficiency [1]. The quality of the anodes is the key to reducing the additional carbon losses. The materials used in anode production are dry aggregate (fresh petroleum coke, butts as well as recycled green and baked anodes) and binder coal tar pitch. The dry aggregate with a known granulometry is mixed under desired mixing conditions (temperature, power, etc.) to make a paste. The paste is then compacted in a vibro-compactor under predetermined operating conditions (amplitude, frequency, charge, temperature, and vacuum) to form green anodes. The green anodes are baked in a furnace using a given heating rate, baking time, etc. to produce baked anodes. Thus, there are several parameters such as raw material properties, granulometry, and process conditions (mixing, forming, and baking) which affect the quality of an anode. Different researchers have been working on the effect of different parameters on anode quality

When there are many parameters influencing a process, the most comprehensive way to study the effect of each parameter is to vary one parameter at a time, keeping all other parameters constant. However, this approach is hardly applicable in the case of carbon anodes. The raw materials are usually nonhomogeneous, and a large number of parameters are inter-related. For example, if different cokes with different porosities are used to study the effect of coke porosity on green anode density, not only the coke porosity changes, but also other coke properties change which, in turn, affect the green anode density. If cokes with different vanadium contents are used to study its effect on anode reactivity, usually the change in vanadium content is accompanied by changes in the levels of other impurities as well as other coke properties such as porosity, etc. It is quite challenging to study experimentally the effect of different parameters on anode quality since the variation of more than one parameter at the same time makes it difficult to carry out a comprehensive analysis of data.

There are different tools for analysis of such data. Different types of graphs such as X vs.Y, bar, histograms etc. are often used; but, they have constraints regarding to the utilization of the number of variables. In addition, the graphical representations sometimes fail to show the trend because of the effect of other parameters. There are statistical tools available such as the analysis of variance (ANOVA), covariance matrix, p-value matrix, etc. to study the effect of different input parameters on the output variables. It may be possible to see trends using these tools; but, sometimes it becomes difficult to quantify their impact. For example, when the p-value matrix is used, a p-value of less than 0.05 usually indicates that the parameter is statistically significant [2], but it does not quantify its effect. Also, these analyses usually require the assumption of linear dependency between the independent and dependent parameters. In addition, ANOVA constricts the comparison of output properties to categorical variables only. This limits the use of ANOVA in data analysis.

Multivariable analysis is an important tool to study the effect of a number of variables on an output parameter. There are different multivariable analysis methods such as linear multivariable analysis (LMA), regression, partial least square (PLS) regression, regression trees, artificial neural network (ANN), genetic algorithms, etc. These are all useful tools, but they also have limitations. At times, they may lead to a false interpretation of the data.

LMA or regression analysis works well if there is some definite mathematical relationship between the independent and dependent variables. The predictor variables also need to be linearly independent. It is difficult to have such a relation in the case of anodes because the dependency is often complex or unknown. The models using the regression and linear multivariable analyses 
usually take shorter time to develop compared ANN and genetic algorithms.

The PLS regression method is a combination of the principal component analysis and the linear multivariable analysis. It uses a linear regression method to find a correlation between predicted and experimental values and simultaneously identifies the components which are significant. The PLS regression method is particularly suited for cases when there are more variables than observations [3]; these cases are difficult to solve with any other tool. Regression tree is another approach for solving a regression problem. The threshold value for dividing a dataset to two or more subsets is determined based on the minimization of the leastsquare regression error. A tree is a hierarchical representation of the data. It does not need any well-defined mathematical relationship, but its drawback is that the threshold does not usually correspond to the optimal boundary for dividing the input parameters at different hierarchical levels [4]. Thus, most of the time, its predictions are not very accurate.

The artificial neural network and genetic algorithms are tools often used in artificial intelligence. The principle for ANN is similar to the functioning of a human brain and is based on pattern recognition and identification. Genetic algorithms use the process of natural selection. They generate solutions for optimization problems using techniques inspired by natural or biological evolution, such as inheritance, mutation, selection, crossover, etc. [5]. However, the genetic algorithms may not be efficient if the complexity is too high. If the number of variables increases, then there is often an exponential increase in the search space size [6]. ANN is a powerful predictive tool and finds application in various fields where it is difficult to have any definite mathematical relationship between the input and the output variables. A feedforward ANN model with sigmoidal transfer function and backpropagation training algorithm is considered as a universal function approximator [7]. The limitations for ANN are the requirement of a large diversity of training data on the problem at hand and the unavailability of any defined rule to develop the model. As anode plants usually maintain a large amount of data over a long period of time, ANN can become a useful tool for the prediction of anode quality.

In this work, three different techniques of the multivariable analysis, namely, LMA, PLS, and ANN, have been applied to the published data of Tkac [8]. The effects of input parameters such as vacuum, vibro-compaction time, green anode density, and heating rate on the baked anode density were studied using the three methods, and the results were compared.

\section{Methodology}

In this study, data published in the literature [8] on the impact of vibro-forming and vibration time on baked anode porosity development have been used. In the reported study, twenty pilot scale anodes with similar composition were made using an industrial paste produced in the paste plant of Hydro-Aluminium. The recipe contained $30 \%$ butts. The aggregate was preheated and mixed with pitch in an industrial kneader at $174^{\circ} \mathrm{C}$. The paste was then transferred to an Eirich mixer. After cooling in the Eirich mixer to $154^{\circ} \mathrm{C}$, the paste was taken out and transported to the laboratory. In the laboratory, the paste was then mixed again in an intensive Eirich laboratory mixer (RV $08 \mathrm{~W}$ ) for 5 minutes at $165^{\circ} \mathrm{C}$. Then, the paste was compacted at $165^{\circ} \mathrm{C}$ and $50 \mathrm{~Hz}$ using four different vibration times $(0.5,1,2,3 \mathrm{~min})$ and two different pressures (one with $4 \mathrm{kPa}$ vacuum and the other at $100 \mathrm{kPa}$ atmospheric pressure without vacuum) above the mold. The green anodes were baked at four different heating rates; $10,35,60$, and $80^{\circ} \mathrm{C} / \mathrm{h}$ followed by 5 -hour soaking time. The densities of the green and baked anodes were measured for all 20 cases. Table 1 summarizes the results of this study.

To prevent the false representation of the impact of different parameters due to large differences in their values (for example, the heating rate varied between 10 and $80^{\circ} \mathrm{C} / \mathrm{h}$ but the green anode density between 1.641 and $1.700 \mathrm{~g} / \mathrm{cc}$ ), all the parameters were normalized as follows:

Normalized value $=\frac{\text { Value to be Normalized }- \text { Minimum Value }}{\text { Maximum Value }- \text { Minimum Value }}$

Then, the effects of different parameters on the baked anode density were studied using LMA, PLS, and ANN; and the results were compared.

Table 1. Baked anode densities corresponding to different input parameters [8]

\begin{tabular}{|c|c|c|c|c|}
\hline $\begin{array}{c}\text { Vacuum } \\
(\text { Yes }=1 ; \\
\text { No }=0)\end{array}$ & $\begin{array}{c}\text { Vibration } \\
\text { time } \\
\text { (min) }\end{array}$ & $\begin{array}{c}\text { Heating } \\
\text { rate } \\
\left({ }^{\circ} \mathrm{C} / \mathrm{h}\right)\end{array}$ & $\begin{array}{c}\text { Green } \\
\text { anode } \\
\text { density } \\
\text { (g/cc) }\end{array}$ & $\begin{array}{c}\text { Baked } \\
\text { anode } \\
\text { density } \\
\text { (g/cc) }\end{array}$ \\
\hline 1 & 3.0 & 10 & 1.700 & 1.619 \\
\hline 0 & 3.0 & 10 & 1.674 & 1.594 \\
\hline 0 & 2.0 & 10 & 1.686 & 1.588 \\
\hline 0 & 1.0 & 10 & 1.660 & 1.586 \\
\hline 0 & 0.5 & 10 & 1.653 & 1.579 \\
\hline 1 & 3.0 & 35 & 1.685 & 1.581 \\
\hline 0 & 3.0 & 35 & 1.673 & 1.550 \\
\hline 0 & 2.0 & 35 & 1.683 & 1.580 \\
\hline 0 & 1.0 & 35 & 1.659 & 1.560 \\
\hline 0 & 0.5 & 35 & 1.650 & 1.545 \\
\hline 1 & 3.0 & 60 & 1.653 & 1.559 \\
\hline 0 & 3.0 & 60 & 1.665 & 1.537 \\
\hline 0 & 2.0 & 60 & 1.677 & 1.554 \\
\hline 0 & 1.0 & 60 & 1.658 & 1.537 \\
\hline 0 & 0.5 & 60 & 1.646 & 1.534 \\
\hline 1 & 3.0 & 80 & 1.641 & 1.546 \\
\hline 0 & 3.0 & 80 & 1.664 & 1.527 \\
\hline 0 & 2.0 & 80 & 1.677 & 1.523 \\
\hline 0 & 1.0 & 80 & 1.655 & 1.528 \\
\hline 0 & 0.5 & 80 & 1.645 & 1.527 \\
\hline
\end{tabular}

Linear multivariable analysis was done using the method described by Bhattacharyay et al. [9]. If the input parameters are $\mathrm{X} 1, \mathrm{X} 2, \ldots, \mathrm{Xn}$ and $\mathrm{Y}$ is the output parameter; then, $\mathrm{Y}$ can be expressed as: 


$$
\mathrm{Y}=\sum_{i=0}^{n}(\mathrm{Ai} * \mathrm{Xi})
$$

where, Ai represents the weighting factor for the input parameter $\mathrm{Xi}$. If the value of $\mathrm{Ai}$ is positive, then an increase in $\mathrm{Xi}$ will increase $\mathrm{Y}$; and if the value of $\mathrm{Ai}$ is negative, then an increase in $\mathrm{Xi}$ will decrease $\mathrm{Y}$. If the value of the weighting factor for a parameter is greater compared to that for another parameter, then the parameter with the greater value of weighting factor will have more influence on the output parameter. A model was developed using Matlab to calculate the coefficients.

PLS analysis was first developed by Wold [10]. It has been applied for data analysis by many researchers [11-14]. In this technique, linear combinations of several predictors on an output variable are analyzed. Associations between the predictor and the output parameters are established by extracting coefficients using the predictor variables that can successfully explain the significance of the output variable most of the time. PLS analysis can be a useful tool when the number of predictor variables is the same or more than the number of observations [3], and there exists a strong correlation between the predictors and the output property. It is also applicable to the cases with more than one output variable. It identifies the weighting factors for each of the variables. The sign and the relative magnitude of the weighting factors indicate the tendencies similar to ones explained above for the linear multivariable analysis. The PLS analysis was carried out using the plsregress function available in the statistical toolbox of Matlab. This function returns the weighting factors for different input parameters for a given set of values of a number of predictors and an output parameter. In the case of LMA and PLS, all 20 data sets (see Table 1 ) were used.

The concept of ANN was first introduced by McCulloch and Pitts in 1943 [15]. It started gaining importance with the advent of back-propagation training algorithm. There are different ANN models such as perceptron [15], feed- forward [16], recurrent [17], radial basis function neural networks [18-20], etc. Out of these different models, feed-forward ANN with back-propagation training is the most widely used one.

In this work, a feed-forward neural network was developed using the neural network toolbox in Matlab. The network had one input and one output layer. The input and the output layers were connected by a hidden layer. The hidden layer can have one or more sub-layers. Usually transfer functions are associated to each of the hidden sub-layers. The role of the transfer function is to convert the input to a hidden layer into values ranging between 0 and 1 . This helps identify the pattern between the input and output variables. In this model, a combination of sigmoidal and linear transfer functions was associated to the hidden layer. The number of neurons associated to the hidden layer was determined based on trial and error. In the ANN model, the weights and bias values are associated to the neurons and the layers. The values are initialized using a random number generator available in Matlab. The seed of the random number generator was chosen based on trial and error. During the training process, the weights and biases are adjusted using a training algorithm. Training is usually done using a certain number of sets of data. In this case, 14 sets of data were used for training. Out of those 14 sets of data, one was used for testing and one was used for validation during the training phase. The testing and validation data sets help prevent the over-training of the ANN model. Due to the small number of data sets available, the choice of data sets for validation and testing was limited. The data sets for training were chosen on a random basis. Levenberg-Marquardt training algorithm (LMTA) was used as the back-propagation training algorithm [9]. The advantage of LMTA is that it combines the steepest descent method and the Gauss-Newton algorithm and therefore inherits the speed advantage of the Gauss-Newton algorithm and the stability of the steepest descent method.

The aim of an ANN model is to understand the pattern in the sets of data used for training and classify them into groups. This process is called learning. Gradient descent learning algorithm was used in the model. The remaining six sets of data were not used for training. They were used to verify the prediction ability of the final model.

The final ANN model was verified using two approaches. The first one is based on the $\mathrm{R}^{2}$ value of the predicted and experimental values using a linear fit, and the second one measures the range of error. First, the baked anode density was predicted for all 20 cases using the input parameters. Then, the predicted values were plotted against the experimental values, and the regression coefficient $\mathrm{R}^{2}$ was calculated for a linear trend line. If the value of $\mathrm{R}^{2}$ is close to 1 , then, it is considered that the model can predict well. In this case, the sets of data which were earlier used for training can improve the $\mathrm{R}^{2}$ value. Therefore, another plot was prepared for the predicted vs. experimental values for only six sets of data which were never used during training. This approach eliminates the effect of the sets of data used earlier for training on the accuracy of the predictions. The $\mathrm{R}^{2}$ value was again calculated. If the value of $\mathrm{R}^{2}$ is close to 1 , then the model is deemed to predict the results well. A good $\mathrm{R}^{2}$ value does not necessarily indicate the error in each of the predictions. The second approach involves the measurement of error between the predicted and experimental values of the output parameter. For the 20 sets of data, if most of the predicted values fall within \pm $0.01 \mathrm{~g} / \mathrm{cc}$, then the model is expected to predict well the unknown cases. The percentage of correct prediction within the specified limit should be ideally close to $100 \%$. If all the verifications are successful for a particular ANN model, that model can be used to predict cases for which no experimental result is available. The developed ANN model was used to study the effect of each parameter on the baked anode density varying only one parameter at a time keeping all other parameters constant.

\section{Results and Discussions}

Figure 1 shows the ANN model used for the analysis. $\mathrm{R}^{2}$ value for the plots of predicted vs. experimental values using 20 points (all) and six points (sets of data not used during training) are 0.93 and 0.94 , respectively. Of the predicted values, $90 \%$ were within a range of $\pm 0.01 \mathrm{~g} / \mathrm{cc}$, and $100 \%$ were within a range of $\pm 0.02 \mathrm{~g} / \mathrm{cc}$ with respect to the experimental baked anode densities. Thus, the model seems capable of predicting the baked anode density for cases for which the experimental results are not available.

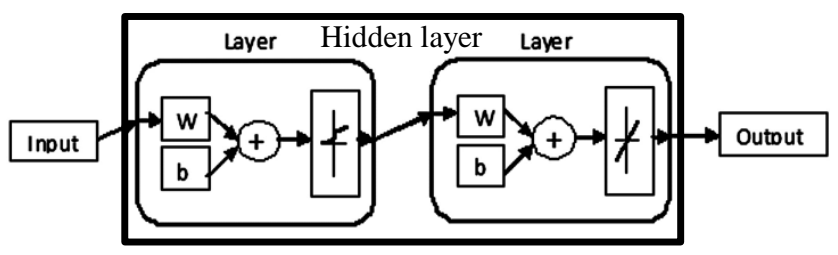

Figure 1. ANN model used 
To study the effect of different parameters, a set of default values were used. The default values were chosen arbitrarily within the range of experimental values. If the input values were chosen outside the range of training data, then the predictionability of the model decreases due to extrapolation. The chosen default values are shown in Table 2.

Table 2. Parameters used for the prediction by ANN.

\begin{tabular}{|l|c|c|c|c|}
\hline $\begin{array}{r}\text { Value of } \\
\text { Parameter } \\
\text { Parameter } \\
\text { studied }\end{array}$ & $\begin{array}{c}\text { Vacuum } \\
\mathbf{( Y e s = 1 /} \\
\mathbf{N o}=\mathbf{0})\end{array}$ & $\begin{array}{c}\text { Vibration } \\
\text { time } \\
\text { (min) }\end{array}$ & $\begin{array}{c}\text { Heating } \\
\text { rate } \\
\left({ }^{\circ} \mathbf{C} / \mathbf{h}\right)\end{array}$ & $\begin{array}{c}\text { Green } \\
\text { anode } \\
\text { density } \\
\text { (g/cc) }\end{array}$ \\
\hline Vacuum & $\mathbf{1 ~ o r ~} \mathbf{0}$ & 1 & 15 & $1.65,1.69$ \\
\hline Vibration time & 1 & Varied & 15 & $1.65,1.69$ \\
\hline Heating rate & 1 & 1 & Varied & $1.65,1.69$ \\
\hline $\begin{array}{l}\text { Green anode } \\
\text { density }\end{array}$ & 1 & $0.5,1$ & 15 & Varied \\
\hline
\end{tabular}

The interpretation of the results obtained by the different methods should be done keeping in mind that whenever the effect of one parameter was studied, all other parameters were kept constant.

Effect of vacuum on baked anode density: Figure 2 presents LMA and PLS predictions and shows the effect of different parameters on the baked anode density. The results show that both models indicate increasing baked anode density with vacuum. The value of the coefficient shows, for both the cases, that vacuum is a significant factor affecting the baked anode density. LMA results imply that vacuum is the most important parameter compared to all other parameters. PLS results show that vacuum is less important compared to the other parameters.

The effect of vacuum on the baked anode density predicted by ANN is presented in Figure 3 for two green anodes with different densities. As can be seen from this figure, ANN demonstrates that the baked anode densities are higher in the presence of vacuum during formation with all other parameters are constant. Published articles describe the importance of vacuum for obtaining good baked anode density. Hulse [21] reported that the presence of vacuum increased the baked anode density by 0.015 g/cc. Application of vacuum helps remove entrapped gas and volatiles from the paste during compaction, which reduces the

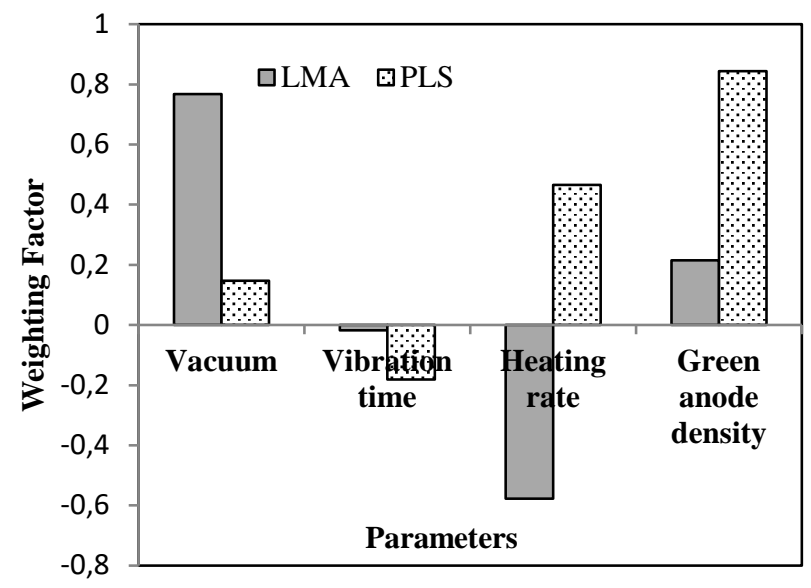

Figure 2. Effect of different parameters on baked anode density using LMA and PLS analyses. chances of pore/crack formation during baking [8] resulting in denser anodes. Thus, from Figures 2 and 3, it can be concluded that LMA, PLS, and ANN identified the expected trend regarding the effect of vacuum on the baked anode density.

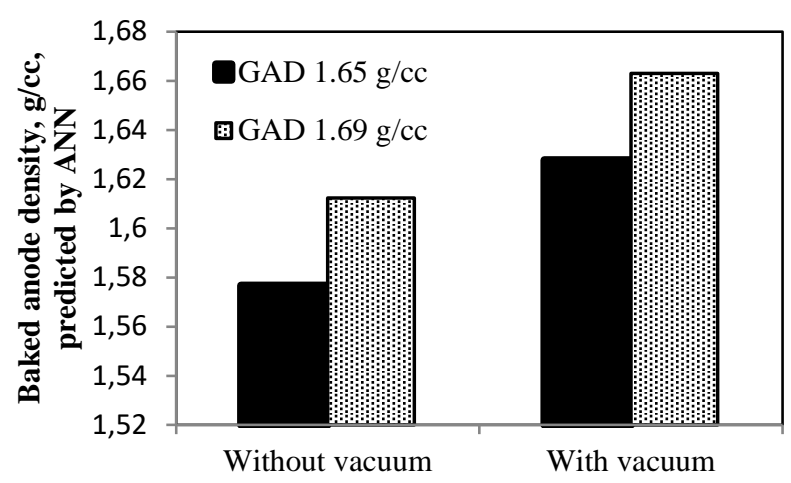

Figure 3. Effect of vacuum on baked anode density (GAD: Green Anode Density)

Effect of vibration time on baked anode density: Figure 2 shows that both LMA and PLS predict a decrease in the baked anode density with increasing vibration time. The vibration time is found to be the least important parameter for LMA whereas it has a significant impact on the baked anode density according to the prediction of PLS. Similarly, the ANN model also predicts a decrease in the baked anode density with increasing vibration time as shown in Figure 4 when the green anode densities and other process parameters are kept constant.

Published articles describe the importance of vibration time on the green anode density. Azari et al. [22] reported that increase in vibration time causes the densification of green anodes. Vibration improves the rearrangement of particles in the mold. When a dense green anode is baked, the volatile evolution causes stress leading to the formation of cracks inside the baked anode. Thus, as can be seen from figures 2 and 4, LMA, PLS, and ANN identified the anticipated trend regarding the effect of vibration time on the baked anode density.

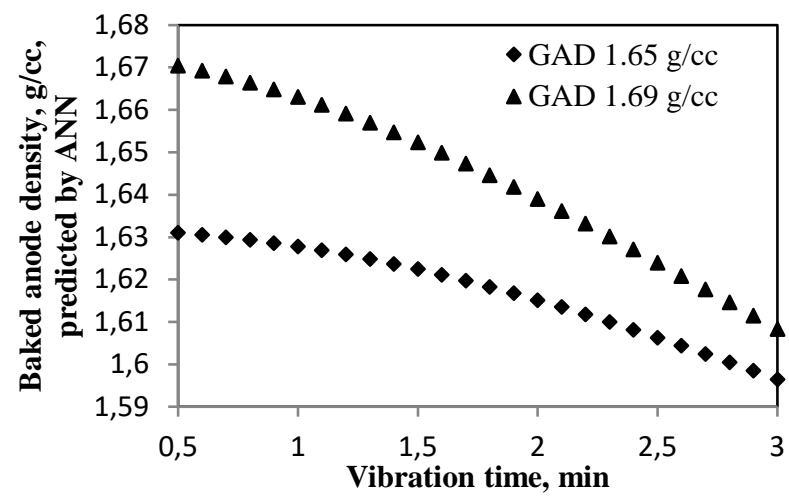

Figure 4. Effect of vibration time on baked anode density.

Figure 4 also shows that at lower vibration times, the effect of the green anode density on the baked anode density is more prominent compared to the same effect at longer vibration times. A variation in the green anode density by $0.04 \mathrm{~g} / \mathrm{cc}$ results in a 
change in the baked anode density by $0.04 \mathrm{~g} / \mathrm{cc}$ at lower vibration times and by only $0.012 \mathrm{~g} / \mathrm{cc}$ at longer vibration times. Thus, ANN shows that the effect of vibration time on baked anode density is more important if the vibration time is low. It is difficult to get such additional information in the case of LMA or PLS.

Effect of heating rate on baked anode density: As shown in Figure 2, LMA predicts that increasing the heating rate decreases the baked anode density. The effect is quite significant. On the other hand, PLS predicts the opposite trend. ANN predictions are given in Figure 5 for green anodes with two different densities. The results show that increase in heating rate decreases the baked anode density. Published articles describe the importance of heating rate on baked anode density. When a green anode is baked, the volatiles are released from the binder pitch. Higher heating rate causes faster release of volatiles and likely to produce more stress and cracks during baking [23], thereby reducing the baked anode density. Thus, from Figures 2 and 5, it can be concluded that LMA and ANN could identify the expected trend regarding the effect of heating rate on the baked anode density. The trend found by PLS is not the right one when compared with the published results.

It can also be seen from Figure 5 that the effect of the green anode density on the baked anode density is more prominent at lower heating rates compared to the effect at higher heating rates. At a low heating rate of $10^{\circ} \mathrm{C} / \mathrm{h}$, a difference of $0.04 \mathrm{~g} / \mathrm{cc}$ in the green anode density resulted in an increase of $0.035 \mathrm{~g} / \mathrm{cc}$ in the baked anode density whereas the same variation in the green anode density at a high heating rate of $80^{\circ} \mathrm{C} / \mathrm{h}$ gave only an increase of $0.004 \mathrm{~g} / \mathrm{cc}$ in the baked anode density. Thus, ANN shows that the effect of heating rate is more significant on the baked anode density if the heating rate is low.

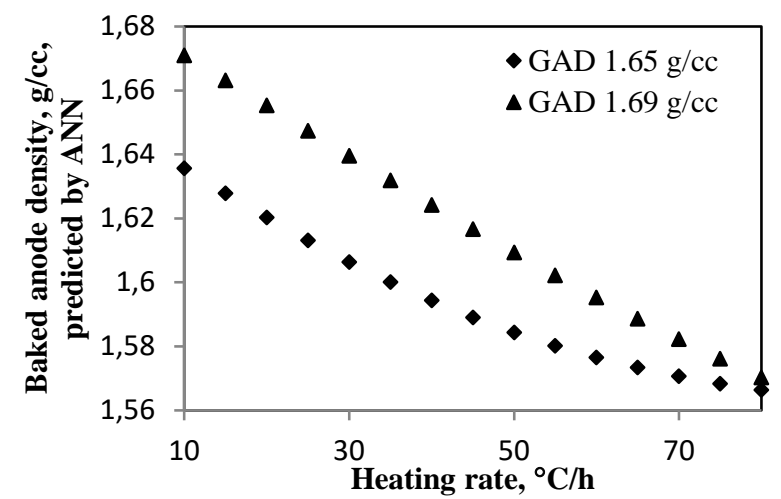

Figure 5. Effect of heating rate on baked anode density.

Effect of green anode density on baked anode density: LMA and PLS both show (see Figure 2) that increase in green anode density increases the baked anode density. PLS shows that the green anode density is the most significant influencing factor whereas LMA shows that it has much less importance. The predictions of ANN at two different vibration times are presented in Figure 6. The ANN model predictions are in line with LMA and PLS and show that increase in green anode density increases the baked anode density. As seen in Figure 6, the increase is significant up to a certain green anode density. Above that, the change in baked anode density is much less pronounced.
Published articles describe the importance of green anode density on baked anode density. Low to medium density of the green anodes facilitates the removal of volatiles and allows the release of stress during baking due to the presence of pores. Denser green anodes do not permit the release of volatiles during baking due to their compactness, resulting in relatively more cracking and thereby reducing the density. When green anode is much denser, the baked anode density is less than expected because of the build-up of the high internal pressure due to the devolatilization of binder pitch during the baking process [8] and the consequent formation of cracks which reduce the baked anode density. Hence, it can be concluded from Figures 2 and 6 that LMA, PLS, and ANN could identify the expected trend concerning the effect of green anode density on the baked anode density.

Figure 6 also shows that the effect of vibration time on baked anode density is less important at low green anode densities compared to the effect at high green anode densities. At low green anode density, when the vibration time increased from 0.5 to 1 min., the baked anode density did not practically vary; however, the baked density varied by $0.008 \mathrm{~g} / \mathrm{cc}$ for the same change in vibration time at high green anode density. Consequently, ANN results show that the effect of green anode density and vibration time on the baked anode density is more significant if the green anode density is high.

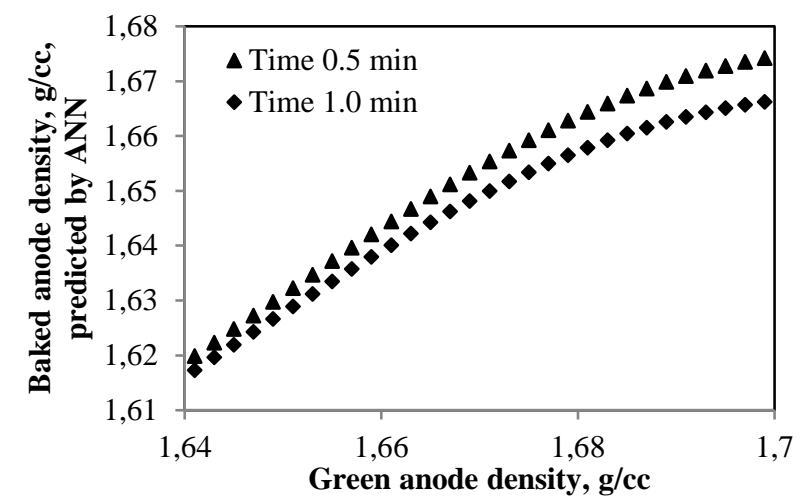

Figure 6. Effect of green anode density on baked anode density.

\section{Conclusions}

Analysis of data using different methods and the comparison of results have always been a challenging task. In this work, published data have been analyzed using three different multivariable analysis techniques, namely LMA, PLS, and ANN. The artificial neural network appears to be a better tool to study the effect of different parameters on anode properties. It can serve as a useful tool to ensure the quality of anodes. The major advantage of ANN over the other methods is that it can efficiently handle highly nonlinear data with noises where there is no existing mathematical relationship, which is usually the case with industrial data.

The development of an efficient ANN model is time consuming because it needs lots of trial and error; but once it is developed, it can predict results for the cases for which there is no experimental data available. ANN, with its power of artificial intelligence, can reduce the number of costly trials necessary to identify the best value for a given parameter in order to improve the anode quality in the aluminum industry. 
LMA and PLS are standard tools for multivariable analysis. Although their predictions were in line with those of ANN for most parameters (vacuum, vibration time, and green anode density), the trend found with PLS for the effect of heating rate was not in agreement with the ANN predictions as well as with those published in the literature. This is most probably due the complex relationship between the anode production parameters and the anode properties.

Even though LMA, PLS, and ANN showed similar trends in many cases, there is a distinct advantage of ANN compared to LMA and PLS. LMA and PLS suggest the impact of different parameters on an output parameter assuming that they are linearly dependent, which may not be the actual case. ANN, on the other hand, can identify the complex relationships between the input and output parameters and can also quantify the magnitude of the impact of different parameters at different conditions. The predictions of ANN are always closer to the real situation compared to those of LMA and PLS.

\section{Acknowledgements}

The technical and financial support of Aluminerie Alouette Inc. as well as the financial support of the National Science and Engineering Research Council of Canada (NSERC), Développement économique Sept-Îles, the University of Québec at Chicoutimi (UQAC), and the Foundation of the University of Québec at Chicoutimi (FUQAC)are greatly appreciated.

\section{References}

[1] D. Kocaefe, A.Sarkar, S. Das, S. Amrani, D. Bhattacharyay, D. Sarkar, Y. Kocaefe, B. Morais, M. Gagnon, Review of different techniques to study the interactions between coke and pitch in anode manufacturing, , TMS Light Metals, Texus, USA, 2013, DOI: 10.1002/9781118663189.ch176

[2] S. Schlotzhauer, Elementary statistics using JMP, SAS Press, Cary, NC: SAS Institute, 2007, 166-169.

[3] J.A. Wegelin, A survey of partial least squares (PLS) methods, with emphasis on the two-block case, Technical report no. 371, Dept. of statistics, University of Washington, 2000, 1-44.

[4] T. Tanaka, S. Ohsuga, M. Ali (Eds.), Industrial and engineering applications of artificial intelligence and expert systems, Proceedings of the Ninth International Conference, Fukuoka, Japan, Gordon and Breach Science Publishers, 1996, 547-552.

[5] M. Mitchell, An introduction to genetic algorithms, MIT Press, USA, 1996.

[6] J.H. Holland, Adaptation in natural and artificial systems, University of Michigan Press, Ann arbour, Michigan, 1975.

[7] H. White, Artificial neural networks: approximation and learning theory. Blackwell, Cambridge, 1992.

[8] M. Tkac, Porosity development in composite carbon materials during heat treatment, $\mathrm{PhD}$ thesis, Norwegian University of Science and Technology, 2007.
[9] D. Bhattacharyay, D. Kocaefe, Y. Kocaefe, B. Morais, M. Gagnon, Application of the artificial neural network (ANN) in predicting anode properties, TMS Light Metals, Texas, USA, John Wiley \& Sons, 2013, DOI: 10.1002/9781118663189.ch201

[10]H. Wold, Models for knowledge: in the making of statisticians, J. Gani (Ed). Springer-Verlag: Berlin, 1982, 89-212.

[11] J.B. Lohmöller, Latent variables path modeling with partial least squares. Physica-Verlag: Heidelberg, 1989.

[12]M. Tenenhausa, V. E. Vinzia, Y.M. Chatelinc, C. Laurob, PLS path modeling, Computational Statistics \& Data Analysis, 2005, 48: 159-205.

[13] G. Russolillo, Partial Least Squares Methods for Non-Metric Data, PhD Thesis, Universit`a degli Studi di Napoli, 2009.

[14] A. M. Ruíz, Patent value models: partial least squares path modeling with mode $\mathrm{C}$ and few indicators, DRUID-DIME Academy Winter $2008 \mathrm{PhD}$ Conference, Universitat Politècnica de Catalunya, 2011.

[15] W.S. McCulloch, W. Pitts, A logical calculus of ideas immanent in nervous activity, Bulletin of Mathematical Biophysics, 1943, 5 : 115 - 133.

[16] M.R. Kaul, L. Hill, C. Walthall, Artificial neural network for corn and soybean yield prediction, Agricultural Systems, 2005, 85(1): 1 - 18.

[17] J.T. Connor, R.D. Martin, L.E. Atlas, Recurrent neural networks and robust times series prediction, IEEE Transactions on Neural Networks, 1994, 5(2): 240 - 254.

[18] Y. Hayashi, J.J.Buckley, E. Czogala, Fuzzy neural network with fuzzy signals and weights, International Joint Conference on Neural Networks, 1992, 2: 696 - 701.

[19]X. Yao, Evolving artificial neural networks, Proceedings of the IEEE, 1999, 87(9): 1423 - 1447.

[20] Z. R. Yang, A novel radial basis function neural network for discriminant analysis, IEEE Transactions on Neural Networks, 2006, 17( 3): 604 - 612.

[21] K. L. Hulse, PhD thesis, R\&D Carbon Ltd., University of Auckland, New Zealand, 2000, ISBN 3-9521028-5-7.

[22] K. Azari, H. Alamdari, D. Ziegler, M. Fafard, Influence of coke particle characteristics on the compaction properties of carbon paste material, Powder Technology, 2014, 257:132-140.

[23] S. Amrani, D. Kocaefe, Y. Kocaefe, B. Morais, G. Blaney, Effect of Heating Rate on the Crack Formation during Baking in Carbon Anodes Used in Aluminum Industry, TMS Light Metals, John Wiley \& Sons, Inc., Hoboken, NJ, USA, 2014, DOI: 10.1002/9781118888438.ch196. 